To appear in the Astrophysical Journal

\title{
Axial instability of rotating relativistic stars
}

\author{
John L. Friedman and Sharon M. Morsink \\ University of Wisconsin-Milwaukee, P.O. Box 413, Milwaukee, WI 53201 \\ friedman@thales.phys.uwm.edu, morsink@pauli.phys.uwm.edu
}

\begin{abstract}
Perturbations of rotating relativistic stars can be classified by their behavior under parity. For axial perturbations (r-modes), initial data with negative canonical energy is found with angular dependence $e^{i m \phi}$ for all values of $m \geq 2$ and for arbitrarily slow rotation. This implies instability (or marginal stability) of such perturbations for rotating perfect fluids. This low $m$-instability is strikingly different from the instability to polar perturbations, which sets in first for large values of $m$. The timescale for the axial instability appears, for small angular velocity $\Omega$, to be proportional to a high power of $\Omega$. As in the case of polar modes, viscosity will again presumably enforce stability except for hot, rapidly rotating neutron stars.

This work complements Andersson's numerical investigation of axial modes in slowly rotating stars.
\end{abstract}

Subject headings: instabilities — relativity — stars: oscillations — stars: rotation

\section{Introduction}

A rotating star is invariant under reflection in the equatorial plane and rotations about the axis of symmetry. It is therefore invariant under parity, the composition of reflection in the equatorial plane and rotation by $\pi$, and perturbations of a rotating star can be chosen to have definite parity. That is, the space of solutions to the Einstein-perfect-fluid equations, linearized about the equilibrium star, is a direct sum of dynamically preserved subspaces with parity eigenvalues \pm 1 . 円

1 It would be natural to call these "odd" or "even" perturbations, but some of the literature in relativistic astrophysics adopts the Regge-Wheeler (1957) terminology, using the term "odd-parity" not 
Perturbations of a spherical star can be divided into two classes, axial and polar, depending on their behavior under parity. Where polar tensor fields on a 2-sphere can be constructed from the scalars $Y_{l}^{m}$, their gradients $\nabla_{A} Y_{l}^{m}$, and the metric $e_{A B}$ on the 2-sphere, axial fields involve the pseudo-tensor $\epsilon_{A B}$, and their behavior under parity is opposite to that of $Y_{l}^{m}$. Thus axial perturbations of odd $l$ are invariant under parity, and axial perturbations with even $l$ change sign. If a mode varies continuously along a sequence of equilibrium configurations that starts with a spherical star and continues along a path of increasing rotation, the mode will be called axial if it is axial for the spherical star. Its parity cannot change along the sequence, but $l$ is well-defined only for modes of the spherical configuration.

Axial perturbations of a spherical stellar model are time-independent convective currents that do not change the density and pressure of the star and do not couple to gravitational waves. Along a continuous sequence of relativistic perfect-fluid models, a mode changes from stable to unstable when its frequency is zero; because they have vanishing frequency in nonrotating models, axial modes could thus be unstable for any model with nonzero rotation. Andersson's numerical work and the computation we present here shows that this is the case: Axial modes for every value of $m$ are unstable for arbitrarily slow rotation.

In spherical stars, gravitational radiation removes positive angular momentum $L_{z}$ from a mode moving in the positive $\phi$ direction and negative angular momentum from a backward-moving mode; and it therefore damps all time-dependent nonaxisymmetric modes. Once the angular velocity of the star is sufficiently large, however, a mode that moves backward relative to the star is dragged forward relative to an inertial observer. Gravitational radiation will then remove positive angular momentum from the mode. But a mode that moves backwards relative to the fluid has negative angular momentum, because the perturbed fluid does not rotate as fast it did without the perturbation. The radiation thus removes positive angular momentum from a mode whose angular momentum is negative. By making the angular momentum of the perturbation increasingly negative, gravitational radiation drives the mode (Friedman and Schutz 1978, Friedman 1978, Chandrasekhar 1970).

For slowly rotating Newtonian stars, the frequencies of axial modes are known (see Sect. 2), and they lie in this unstable regime. In general relativity, Andersson (1997) numerically computes the real part of the frequency of these modes in a slow-rotation

for a perturbation that changes sign under parity but for an axial perturbation, whose behavior is opposite to that of $Y_{l}^{m}$. 
approximation, while we show analytically that that their canonical energy can be made negative; the fact that they are unstable (or marginally stable) follows analytically from this and numerically from Andersson's frequency.

Formal work on the stability of rotating stars does not distinguish between axial and polar modes, but explicit studies of nonaxisymmetric instability of stellar modes had been restricted to polar perturbations, for which the onset of instablity is very different. There are unstable polar modes with arbitrarily small rotation, but as $\Omega \rightarrow 0$, the minimum $m$ for which a mode is unstable grows without bound.

In Sect. 2, we consider the stability of axial perturbations in the Newtonian limit, showing that for any nonzero $\Omega$ the canonical energy can be made negative for each

$m \geq 2$. The perturbations we consider have angular behavior $e^{i m \phi}$ and parity $(-1)^{m+1}$, corresponding to an $l=m$ mode of a corresponding sperical star. In Sect. 3, we extend the computation to general relativity, again finding initial data with negative canonical energy. Finally, in Sect. 4, we estimate the timescale and briefly discuss implications and future problems.

Spacetime indices will be lower-case Greek letters, spatial indices lower-case Latin. We will work in gravitational units, setting $G=c=1$.

\section{Newtonian stars}

We consider a uniformly rotating, axisymmetric, self-gravitating perfect fluid, described by a gravitational potential $\nu$, density $\rho$, pressure $p$ and 3 -velocity

$$
v^{a}=\Omega \phi^{a} \text {. }
$$

These satisfy the equilibrium equations

$$
\begin{gathered}
\nabla^{2} \nu=4 \pi \rho \\
\nabla_{a}\left(h-\frac{1}{2} v^{2}+\nu\right)=0,
\end{gathered}
$$

together with an equation of state of the form

$$
\rho=\rho(p) .
$$

Here $h$ is the specific enthalpy in a comoving frame,

$$
h=\int \frac{d p}{\rho} .
$$


We will work in the Lagrangian formalism (Friedman and Schutz 1978a) describing perturbations in terms of a Lagrangian displacement $\xi^{a}$ that connects fluid elements in the equilibrium and perturbed star. The Eulerian change $\delta Q$ in a quantity $Q$ is related to its Lagrangian change $\Delta Q$ by

$$
\Delta Q=\delta Q+£_{\xi} Q
$$

where $£_{\xi}$ is the Lie derivative along $\xi^{a}$.

The displacement $\xi^{a}$ determines the fluid perturbation:

$$
\begin{gathered}
\Delta v^{a}=\partial_{t} \xi^{a}, \\
\frac{\Delta p}{\gamma p}=\frac{\Delta \rho}{\rho}=-\nabla_{a} \xi^{a} .
\end{gathered}
$$

For angular dependence $e^{i m \phi}$, we have the corresponding Eulerian changes

$$
\begin{aligned}
\delta v^{a} & =\left(\partial_{t}+i m \Omega\right) \xi^{a} \\
\delta \rho & =-\nabla_{a}\left(\rho \xi^{a}\right) \\
\delta p & =\frac{d p}{d \rho} \delta \rho
\end{aligned}
$$

and the change in the potential is given by

$$
\nabla^{2} \delta \nu=4 \pi \delta \rho
$$

An axial perturbation of a spherical Newtonian star is a change in the fluid's velocity of the form

$$
\delta v^{a}=\tilde{\zeta}(r) \epsilon^{a b c} \nabla_{b} r \nabla_{c} Y_{l}^{m} .
$$

That is, it is a vector with arbitrary $r$-dependence, whose behavior on a symmetry two-sphere is given by $\epsilon^{A B} \nabla_{B} Y_{m}^{l}$. P

The time independence of the perturbation can be seen as follows. An axial displacement vector has the form

$$
\xi^{a}=\zeta(r, t) \epsilon^{a b c} \nabla_{b} r \nabla_{c} Y_{l}^{m}
$$

\footnotetext{
${ }^{2}$ One can regard a tensor on the two-sphere as a tensor on $R^{3}$ that is is orthogonal in all of its indices to $\nabla_{a} r$ and is Lie derived by $\nabla^{a} r$. The antisymmetric tensor $\epsilon_{A B}$ associated with the unit metric $e_{A B}$ on the two-sphere is then identified with $\epsilon_{a b}=\epsilon_{a b c} \nabla^{a} r$, and $\epsilon^{A B} \nabla_{B} Y_{l}^{m}$ is identified with $\epsilon^{a b} \nabla_{b} Y_{l}^{m}$.
} 
The perturbed scalars $(\delta p, \delta \rho, \delta \nu)$ must vanish, because they are proportional to $Y_{l}^{m}$ in an $(l, m)$ representation and thus cannot be axial. Explicitly, from the relations

$$
\nabla_{a} \xi^{a}=0, \quad \xi^{a} \nabla_{a} \rho=0
$$

it follows that the Eulerian change in $\rho$-and hence in $p$ and $\nu$-vanishes. Then only the velocity changes, with

$$
\delta v^{a}=\partial_{t} \xi^{a}
$$

and the perturbed Euler equation,

$$
\delta\left[\left(\partial_{t}+£_{v}\right) v_{a}+\nabla_{a}\left(h-\frac{1}{2} v^{2}+\nu\right)\right]=0,
$$

becomes simply

$$
\partial_{t} \delta v^{a}=0
$$

Thus for a spherical Newtonian star, axial perturbations are time-independent changes of the velocity, having the form (13).

We will shortly derive the axial instability in a way that does not rely on normal modes. Let us first however note that the known frequency of axial modes suffices to imply their instability for slowly rotating models.

For a slowly rotating star, the frequency $\sigma$ of these modes is no longer zero. Instead, conservation of circulation implies in the Newtonian limit (Papaloizou and Pringle 1978) the relation

$$
\sigma+m \Omega=\frac{2 m \Omega}{l(l+1)},
$$

where the perturbation's time dependence is $e^{i \sigma t}$.

Eq. (19) is a consequence of conservation of circulation, the curl of Eq. (17):

$$
\left(\partial_{t}+i m \Omega\right) \epsilon^{a b c} \nabla_{b} \Delta v_{c}=0
$$

(To obtain this equation, note that in Eq. (17) $\delta$ can be replaced by $\Delta$, because the equation is satisfied by the equilibrium configuration.) For $\xi^{a}$ of the form (14), we obtain

$$
\begin{aligned}
\epsilon^{a b c} \nabla_{b} \Delta v_{c} \nabla_{a} r & =\left(\partial_{t}+\Omega \partial_{\phi}\right) \epsilon^{r \theta \phi}\left(\partial_{\theta} \xi_{\phi}-\partial_{\phi} \xi_{\theta}\right)-2 \Omega \sin \theta \xi^{\theta} \\
& =i[(\sigma+m \Omega) l(l+1)-2 m \Omega] \frac{\zeta}{r^{2}} Y_{l}^{m} .
\end{aligned}
$$

Eq. (21) then has as a solution

$$
\sigma=-m \Omega\left[1-\frac{2}{l(l+1)}\right]
$$


The frequency $\sigma$ is negative when $l \geq 2$ while the frequency $\sigma+m \Omega$ relative to a comoving observer is positive (Eq. 19). Because modes that travel backwards relative to the star, are pulled forwards relative to an inertial frame, they are formally unstable for arbitrarily small values of the star's rotation.

Stability is governed by the sign of the canonical energy $E_{c}$, when expressed in terms of canonical displacements $\xi^{a}$ (Friedman and Schutz 1978). That is, a model is unstable to perturbations with angular dependence $e^{i m \phi}$ when there is initial data with this angular behavior for which $E_{c}(\xi)<0$. The canonical energy has the form

$$
\begin{aligned}
E_{c}= & \frac{1}{2} \int\left[\rho|\dot{\xi}|^{2}-\rho|v \cdot \nabla \xi|^{2}+\gamma p|\nabla \cdot \xi|^{2}\right. \\
& +(\bar{\xi} \cdot \nabla p \nabla \cdot \xi+\xi \cdot \nabla p \nabla \cdot \bar{\xi} \\
& \left.+\xi^{a} \xi^{b}\left(\nabla_{a} \nabla_{b} p+\rho \nabla_{a} \nabla_{b} \nu\right)-\frac{1}{4 \pi}|\nabla \delta \nu|^{2}\right] d V .
\end{aligned}
$$

We evaluate it to order $\Omega^{2}$.

For a slowly rotating star,

$$
\rho=\rho_{0}+O\left(\Omega^{2}\right), \rho=p_{0}+O\left(\Omega^{2}\right), \nu=\nu_{0}+O\left(\Omega^{2}\right),
$$

with $\rho_{0}, p_{0}$ and $\nu_{0}$ values for the corresponding spherical star with the same mass. Then for $\xi^{a}$ of the form (14), we have

$$
\xi \cdot \nabla \rho=O\left(\Omega^{2}\right), \quad \xi \cdot \nabla p=O\left(\Omega^{2}\right), \quad \xi \cdot \nabla \nu=O\left(\Omega^{2}\right),
$$

and

$$
E_{c}=\frac{1}{2} \int d V\left[\rho|\dot{\xi}|^{2}-\rho|v \cdot \nabla \xi|^{2}+\xi^{a} \xi^{b}\left(\nabla_{a} \nabla_{b} p+\rho \nabla_{a} \nabla_{a} \nu\right)\right]+O\left(\Omega^{4}\right)
$$

In the final term,

$$
\begin{aligned}
\nabla_{a} \nabla_{b} p+\rho \nabla_{a} \nabla_{b} \nu & =\rho \nabla_{a}\left(\frac{1}{\rho} \nabla_{b} p+\nabla_{b} \nu\right)+\frac{1}{\rho} \nabla_{a} \rho \nabla_{b} p \\
& =\frac{1}{2} \rho \nabla_{a} \nabla_{b} v^{2}+\frac{1}{\rho} \nabla_{a} \rho \nabla_{b} p
\end{aligned}
$$

where the equation of hydrostatic equilibrium, (3), is used to obtain the last equality. Then

$$
\bar{\xi}^{a} \xi^{b}\left(\nabla_{a} \nabla_{b} p+\rho \nabla_{a} \nabla_{b} \nu\right)=\frac{1}{2} \rho \Omega^{2} \bar{\xi}^{a} \xi^{b} \nabla_{a} \nabla_{b}\left(\varpi^{2}\right),
$$

with $\varpi=r \sin \theta$ the distance from the symmetry axis. 
After a straightforward computation, we find

$$
E_{c}=\frac{1}{2} \int d V \rho\left[|\dot{\xi}|^{2}-m^{2} \Omega^{2}|\xi|^{2}+4 m \Omega^{2} r^{2} \sin \theta\left|\cos \theta \xi^{\theta} \xi^{\phi}\right|\right] .
$$

An initial data set $(\xi, \dot{\xi})$ is canonical if it satisfies

$$
q^{a} \equiv \epsilon^{a b c} \nabla_{b} \Delta_{\xi} v_{c}=0
$$

Writing $v^{a}=\Omega \phi^{a}$ for the equilibrium star and $z^{a}$ for the unit vector $\nabla^{a} z$, we obtain

$$
q^{a}=\left(\partial_{t}+\Omega £_{\phi}\right) \epsilon^{a b c} \nabla_{b} \xi_{c}-2 \Omega £_{z} \xi^{a}
$$

In a cylindrical chart $\varpi, z, \phi$, the components of $q^{a}$ are

$$
q^{i}=\left(\partial_{t}+\Omega \partial_{\phi}\right) \frac{1}{\varpi}\left(\partial_{j} \xi_{k}-\partial_{k} \xi_{j}\right)-2 \Omega \partial_{z} \xi^{i}, \quad i, j, k \text { cyclic. }
$$

To show the existence of canonical initial data that makes $E_{c}$ negative for each $m$ we specialize to the case $l=m$. A displacement $\xi^{a}$ given by eq. (14) with $l=m$ will be canonical if for suitable $\sigma$,

$$
\zeta(r) \propto r^{m+1}, \quad \partial_{t} \zeta=i \sigma \zeta
$$

We can choose

$$
\begin{aligned}
\xi^{\varpi} & =i \varpi^{m-1} z e^{i m \phi} \\
\xi^{z} & =-i \varpi^{m} e^{i m \phi} \\
\xi^{\phi} & =-\varpi^{m-2} z e^{i m \phi} .
\end{aligned}
$$

We then have

$$
\begin{aligned}
q^{\varpi} & =i[(\sigma+m \Omega)(m+1)-2 m \Omega] \varpi^{m-1} e^{i m \phi} \\
q^{z} & =0 \\
q^{\phi} & =-[(\sigma+m \Omega)(m+1)-2 m \Omega] \varpi^{m-2} e^{i m \phi},
\end{aligned}
$$

and $q^{a}=0$, when

$$
\sigma=-m \Omega\left[1-\frac{2}{m(m+1)}\right] .
$$

The agreement with Eq. (22) reflects the fact that the displacement vector of a normal mode is canonical. 
Armed with $\xi^{a}$, we can verify that the canonical energy is negative. From (34 36) we have,

$$
|\xi|^{2}=\frac{\zeta^{2}}{r^{2}} \sin ^{2(m-1)} \theta\left(1+\cos ^{2} \theta\right),
$$

and

$$
r^{2} \sin \theta \cos \theta\left|\xi^{\theta} \xi^{\phi}\right|=\frac{\zeta^{2}}{r^{2}} \sin ^{2(m-1)} \theta \cos ^{2} \theta .
$$

Then, explicitly performing the $\theta$ integration, we find that the canonical energy of Eq. (29) is given by

$$
E_{c}=-\frac{1}{2} \int d V \rho|\xi|^{2}\left[(m \Omega-\sigma)(m \Omega+\sigma)-\frac{2 m}{2 m+1} \Omega^{2}\right],
$$

implying

$$
E_{c}<0,
$$

for $\sigma$ satisfying Eq. (19).

For a normal mode, the canonical condition is equivalent to the curl of the perturbed Euler equations. In fact, the displacement $\xi^{a}$ given by Eqs. (34 36) (or, equivalently, by Eqs. (14) and (33)) satisfies the full Euler equations and is the correct form of an $l=m$ axial mode up to terms of order $\Omega^{3}$.

\section{Relativistic stars}

The spacetime of a uniformly rotating relativistic star is described, to lowest order in angular velocity, by a metric

$$
d s^{2}=-e^{2 \nu} d t^{2}+e^{2 \psi}(d \phi-\omega d t)^{2}+e^{2 \lambda} d r^{2}+e^{2 \mu} d \theta^{2},
$$

with commuting Killing vectors $t^{\alpha}$ and $\phi^{\alpha}$. The fluid has four-velocity

$$
u^{\alpha}=u^{t}\left(t^{\alpha}+\Omega \phi^{\alpha}\right),
$$

and its energy density $\epsilon$, pressure $p$, and specific entropy $s$, satisfy an equation of state,

$$
\epsilon=\epsilon(p, s)
$$

(neutron stars are effectively isentropic, with no dependence on $s$ ). The field equations

$$
G_{\alpha \beta}=8 \pi T_{\alpha \beta}
$$

imply the equation of hydrostatic equilibrium,

$$
\nabla_{\alpha}\left(\tilde{h}+\log u^{t}\right)=0,
$$


where

$$
\tilde{h}=\int \frac{d p}{\epsilon+p} .
$$

As in the Newtonian limit, an axial perturbation of a spherical relativistic star involves a time-independent change in the fluid's 4-velocity, and no change in the star's pressure or density. (The metric, however, does change, as will be discussed below in the treatment of initial data describing a perturbed rotating star.) The change in the 4-velocity has the form

$$
\delta u^{\alpha}=\zeta(r) \epsilon^{\alpha \beta \gamma \delta} \nabla_{\beta} t \nabla_{\gamma} r \nabla_{\delta} Y_{m}^{l},
$$

where

$$
\epsilon^{\alpha \beta}:=\epsilon^{\alpha \beta \gamma \delta} u_{\gamma} r_{\delta},
$$

with

$$
r_{\alpha}=e^{\lambda} \nabla_{\alpha} r
$$

For a rotating star, the frequency $\sigma$ of these modes is no longer zero. To show that axial perturbations with arbitrary values of $m$ are unstable for slowly rotating perfect-fluid models, we shall again consider perturbations corresponding to a Lagrangian displacement with initial data of the form

$$
\begin{gathered}
\xi^{\alpha}=\zeta(r) \epsilon^{\alpha \beta} \nabla_{\beta} Y_{m}^{l} \\
£_{\mathbf{t}} \xi^{\alpha}=i \sigma(r) \xi^{\alpha} .
\end{gathered}
$$

The dependence of $\sigma$ on $r$ arises because it is easier to work with initial data that is not data for a pure outgoing mode.

Initial data for the perturbed star and geometry is a set $\left(\xi^{\alpha}, £_{\mathbf{t}} \xi^{\alpha}, h_{\alpha \beta}, £_{\mathbf{t}} h_{\alpha \beta}\right)$, where $h_{\alpha \beta}=\delta g_{\alpha \beta}$, satisfying the initial value equations,

$$
\delta\left(G^{\alpha \beta}-8 \pi T^{\alpha \beta}\right) \nabla_{\beta} t=0,
$$

and the equations governing a canonical displacement. Given such a set, we have (see Friedman and Ipser 1992 for a review of the perturbation formalism used here)

$$
\begin{aligned}
\Delta g_{\alpha \beta} & =h_{\alpha \beta}+2 \nabla_{(\alpha} \xi_{\beta)}, \\
\Delta u^{\alpha} & =\frac{1}{2} u^{\alpha} u^{\beta} u^{\gamma} \Delta g_{\beta \gamma},
\end{aligned}
$$

and

$$
\frac{\Delta p}{\gamma p}=\frac{\Delta \epsilon}{\epsilon+p}=\frac{\Delta n}{n}=-\frac{1}{2} q^{\alpha \beta} \Delta g_{\alpha \beta} .
$$


A spherical star has metric

$$
d s^{2}=-e^{2 \nu} d t^{2}+e^{2 \lambda} d r^{2}+r^{2}\left(d \theta^{2}+\sin ^{2} \theta d \phi^{2}\right)
$$

and four-velocity

$$
u^{\alpha}=e^{-\nu} t^{\alpha}
$$

Axial perturbations of the metric can have nonzero vector contributions

$$
h_{\alpha \beta} t^{\beta}, h_{\alpha \beta} r^{\beta}
$$

proportional to the vector

$$
\epsilon_{\alpha}^{\beta} \nabla_{\beta} Y_{l}^{m}
$$

and a nonzero tensor contribution proportional to

$$
\epsilon_{(\alpha}^{\gamma} \nabla_{\beta)} \nabla_{\gamma} Y_{l}^{m}
$$

The form of $\xi^{\alpha}$ implies

$$
\begin{gathered}
\Delta u^{\alpha}=0, \\
\delta u^{\alpha}=-e^{-\nu} £_{\mathbf{t}} \xi^{\alpha},
\end{gathered}
$$

and

$$
\delta \epsilon=\delta p=0
$$

A slowly rotating star has metric for which $\omega$ is $O(\Omega)$ and the remaining departure from spherical is $O\left(\Omega^{2}\right)$ :

$$
d s^{2}=-e^{2 \nu}(1+2 h) d t^{2}+r^{2}(1+2 k) \sin ^{2} \theta(d \phi-\omega d t)^{2}+e^{2 \lambda}(1+2 \ell) d r^{2}+r^{2}(1+2 k) d \theta^{2}
$$

we will need only the fact that $h, k, \ell$ are $O\left(\Omega^{2}\right)$, not their explicit values (Hartle 1967). For a displacement of the form (54), we have

$$
\begin{gathered}
\Delta u^{\alpha}=O\left(\Omega^{2}\right) \\
\delta \epsilon=O\left(\Omega^{2}\right), \quad \delta p=O\left(\Omega^{2}\right)
\end{gathered}
$$

The condition governing a canonical displacement is again related to conservation of circulation, which has the relativistic form (Friedman 1978)

$$
£_{\mathbf{u}} \omega_{\alpha \beta}=0
$$


where $\omega_{\alpha \beta}$ is the relativistic vorticity,

$$
\omega_{\alpha \beta}=2 \nabla_{[\alpha}\left(\frac{\epsilon+p}{n} u_{\beta]}\right)
$$

Then canonical initial data satisfies

$$
\Delta \omega_{\alpha \beta}=0
$$

Now

$$
\Delta \omega_{\alpha \beta}=2 \nabla_{[\alpha}\left(\frac{\epsilon+p}{n} \Delta u_{\beta]}\right)+O\left(\Omega^{2}\right)
$$

with

$$
\begin{aligned}
\Delta u_{\alpha} & =\Delta\left(g_{\alpha \beta} u^{\beta}\right)=\Delta g_{\alpha \beta} u^{\beta}+O\left(\Omega^{2}\right) \\
& =2 \nabla_{(\alpha} \xi_{\beta)} u^{\beta}+O\left(\Omega^{2}\right) .
\end{aligned}
$$

As in the Newtonian case, we will use data with $l=m$, writing $\xi^{\alpha}$ in the form

$$
\begin{gathered}
\xi^{\theta}=i \sin ^{m-1} \theta e^{i m \phi} \frac{\zeta(r, t)}{r^{2}} \\
\xi^{\phi}=-\sin ^{m-2} \theta e^{i m \phi} \frac{\zeta(r, t)}{r^{2}} \cos \theta \\
£_{\mathbf{t}} \xi=i \sigma \xi .
\end{gathered}
$$

From Eq. (74), we obtain

$$
\begin{aligned}
\Delta \omega_{\theta \phi}= & 2 \partial_{[\theta}\left(\frac{\epsilon+p}{n} \Delta u_{\phi]}\right)=0: \\
& u^{t} \sin ^{m+1} \theta e^{i m \phi} \zeta[-i m(m+1)(\sigma+m \Omega)+2 i m(\Omega-\omega)]=0,
\end{aligned}
$$

satisfied when

$$
\sigma+m \Omega=\frac{2 m(\Omega-\omega)}{m(m+1)} .
$$

Thus, as noted earlier, we have not supplied data for a normal mode, but data for which $\sigma$ is a function of $r$, with

$$
\partial_{r} \sigma=-\frac{2}{m+1} \partial_{r} \omega
$$

The remaining components of the canonical condition (73) are

$$
\begin{aligned}
& \Delta \omega_{r \phi}=0: \\
& \partial_{r} \log \left(\frac{\epsilon+p}{n}\right) \Delta u_{\phi}+\partial_{r}\left(\Delta u_{\phi}\right)-\partial_{\phi}\left(\Delta u_{r}\right)=0
\end{aligned}
$$


and

$$
\begin{aligned}
& \Delta \omega_{r \theta}=0: \\
& \partial_{r} \log \left(\frac{\epsilon+p}{n}\right) \Delta u_{\theta}+\partial_{r}\left(\Delta u_{\theta}\right)-\partial_{\theta}\left(\Delta u_{r}\right)=0
\end{aligned}
$$

Using the equation of hydrostatic equilibrium in the form

$$
\partial_{r}\left(\frac{\epsilon+p}{n u^{t}}\right)=0
$$

one obtains from the remaining components of the canonical condition a single equation for $\zeta$

$$
\partial_{r} \zeta \frac{2(\Omega-\omega)}{m+1}+\zeta\left[-\frac{2}{m+1} \partial_{r} \omega-\frac{1}{r^{2}} \partial_{r}\left(r^{2}(\Omega-\omega)\right)-2 \partial_{r} \nu\left(\frac{2(\Omega-\omega)}{m+1}-(\Omega-\omega)\right)\right]=0,
$$

with solution,

$$
\zeta=(\Omega-\omega)^{-1+(m+1) / 2} r^{m+1} \exp [-(m-1) \nu]
$$

We have obtained a canonical displacement, but must still satisfy the initial value equations. We will do so by choosing a metric perturbation for which $h_{\alpha \beta}=0, £_{\mathbf{t}} h_{\alpha \beta} \neq 0$. The initial value equations can be obtained from the action for the perturbation equations, which we will use in any event to write down the canonical energy:

$$
I=\int d^{4} x \sqrt{-g} \mathcal{L}(\bar{\xi}, \bar{h} ; \xi, h)
$$

where

$$
\begin{aligned}
\mathcal{L}(\bar{\xi}, \bar{h} ; \xi, h)=\quad U^{\alpha \beta \gamma \delta} \nabla_{\alpha} \bar{\xi}_{\beta} \nabla_{\gamma} \xi_{\delta}+V^{\alpha \beta \gamma \delta}\left(\bar{h}_{\alpha \beta} \nabla_{\gamma} \xi_{\delta}+h_{\alpha \beta} \nabla_{\gamma} \bar{\xi}_{\delta}\right) \\
\quad-\frac{1}{32 \pi} \epsilon^{\alpha \gamma \epsilon \eta} \epsilon^{\beta \delta \zeta} \nabla_{\gamma} \bar{h}_{\alpha \beta} \nabla_{\delta} h_{\epsilon \zeta} \\
-T^{\alpha \beta} R_{\alpha \gamma \beta \delta} \bar{\xi}^{\gamma} \xi^{\delta}+\left(\frac{1}{2} W^{\alpha \beta \gamma \delta}-\frac{1}{16 \pi} G^{\alpha \beta \gamma \delta}\right) \bar{h}_{\alpha \beta} h_{\gamma \delta} \\
-\frac{1}{2} \nabla_{\gamma} T^{\alpha \beta}\left(\bar{h}_{\alpha \beta} \xi^{\gamma}+h_{\alpha \beta} \bar{\xi}^{\gamma}\right),
\end{aligned}
$$

with

$$
\begin{gathered}
G^{\alpha \beta \gamma \delta}=\frac{1}{2} R^{\alpha(\gamma \delta) \beta}+\frac{1}{4}\left(2 R^{\alpha \beta} g^{\gamma \delta}+2 R^{\gamma \delta} g^{\alpha \beta}-3 R^{\alpha(\gamma} g^{\delta) \beta}-3 R^{\beta(\gamma} g^{\delta) \alpha}\right) \\
+\frac{1}{4} R\left(g^{\alpha \gamma} g^{\beta \delta}+g^{\alpha \delta} g^{\beta \gamma}-g^{\alpha \beta} g^{\gamma \delta}\right) \\
U^{\alpha \beta \gamma \delta}=(\epsilon+p) u^{\alpha} u^{\gamma} q^{\beta \delta}+p\left(g^{\alpha \beta} g^{\gamma \delta}-g^{\alpha \delta} g^{\beta \gamma}\right)-\gamma p q^{\alpha \beta} q^{\gamma \delta}
\end{gathered}
$$


and

$$
2 V^{\alpha \beta \gamma \delta}=(\epsilon+p)\left(u^{\alpha} u^{\gamma} q^{\beta \delta}+u^{\beta} u^{\gamma} q^{\alpha \delta}-u^{\alpha} u^{\beta} q^{\gamma \delta}\right)-\frac{1}{2} \gamma p q^{a \beta} q^{\gamma \delta}
$$

The perturbed initial value equations are given by

$$
0=\frac{\delta I}{\delta h_{\alpha \beta}} \nabla_{\beta} t
$$

When $h_{\alpha \beta}$ vanishes on the intial data surface, the Hamiltonian constraint vanishes. We choose $\dot{h}_{\alpha \beta}$ to have no vector contribution, writing

$$
\dot{h}_{\alpha \beta}=h(r) \epsilon_{(\alpha}^{\gamma} \nabla_{\beta)} \nabla_{\gamma} Y_{l}^{m}
$$

The momentum constraint then has, to $O(\Omega)$, the form

$$
\frac{1}{2}(\epsilon+p) u^{t} £_{\mathbf{u}} \xi^{\alpha}-\frac{1}{32 \pi} \epsilon^{t \alpha \gamma \zeta} \epsilon_{\zeta}^{t \beta \delta} \nabla_{\beta} \dot{h}_{\gamma \delta}=0
$$

or

$$
i(\sigma+m \Omega)(\epsilon+p)\left(u^{t}\right)^{2} \frac{\zeta}{r^{2}}+\frac{1}{32 \pi}(m+1)(m+2) \frac{e^{-2 \nu}}{r^{4}} h=0 .
$$

Thus the initial value equations are satisfied by choosing

$$
h(r)=-32 \pi i \frac{\sigma+m \Omega}{(l-1)(l+2)} r^{2}(\epsilon+p) \zeta
$$

We can now compute the canonical energy, whose relativistic form is

$$
E_{c}=\int_{S} d S_{\epsilon}\left[U^{\epsilon \beta \gamma \delta} \dot{\xi}_{\beta} \nabla_{\gamma} \xi_{\delta}+V^{\gamma \delta \epsilon \beta} h_{\gamma \delta} \xi_{\beta}-\frac{1}{32 \pi} \epsilon^{\epsilon \gamma \rho \zeta} \epsilon^{\beta \delta \eta}{ }_{\zeta} \dot{h}_{\gamma \delta} \nabla_{\beta} h_{\rho \eta}-t^{\epsilon} \mathcal{L}\right]
$$

For a displacement having the axial form (54), we obtain the simpler expression

$$
\begin{aligned}
E_{c}= & \int d V e^{\nu}\left[U^{t \beta \gamma \delta} £_{\mathbf{t}} \bar{\xi}_{\beta} \nabla_{\gamma} \xi_{\delta}-\frac{1}{64 \pi} \epsilon^{t \gamma \delta \zeta} \epsilon^{t \delta \eta} \zeta^{£_{\mathbf{t}}} \bar{h}_{\gamma \delta} £_{\mathbf{t}} h_{\epsilon \eta}\right. \\
& \left.-\frac{1}{2} U^{\alpha \beta \gamma \delta} \nabla_{\alpha} \bar{\xi}_{\beta} \nabla_{\gamma} \xi_{\delta}+\frac{1}{2} T^{\alpha \beta} R_{\alpha \gamma \beta \delta} \bar{\xi}^{\gamma} \xi^{\delta}\right] \\
\equiv & \int d V\left[\mathcal{E}_{f 1}+\mathcal{E}_{f 2}+\mathcal{E}_{f 3}+\mathcal{E}_{g}\right]
\end{aligned}
$$

where the terms $\mathcal{E}_{f i}$ indicate contributions from the fluid, $\mathcal{E}_{g}$ the contribution from the perturbed metric. (This split into fluid and metric is gauge-dependent). Evaluating each term, we obtain

$$
\mathcal{E}_{f 1}=U^{t \beta \gamma \delta} £_{\mathbf{t}} \bar{\xi}_{\beta} \nabla_{\gamma} \xi_{\delta}=-i \sigma(\epsilon+p) u^{t} \bar{\xi}_{\beta} u^{\gamma} \nabla_{\gamma} \xi^{\beta}+O\left(\Omega^{4}\right)
$$




$$
\begin{aligned}
\mathcal{E}_{f 2}=-\frac{1}{2} U^{\alpha \beta \gamma \delta} \nabla_{\alpha} \bar{\xi}_{\beta} \nabla_{\gamma} \xi_{\delta}=- & \frac{1}{2}(\epsilon+p)|u \cdot \nabla \xi|^{2}+\frac{1}{2} p \nabla_{\alpha} \bar{\xi}_{\beta} \nabla^{\beta} \xi^{\alpha} \\
& +O\left(\Omega^{4}\right) . \\
\mathcal{E}_{f 3}=\frac{1}{2} T^{\alpha \beta} R_{\alpha \gamma \beta \delta} \bar{\xi}^{\gamma} \xi^{\delta}=- & \frac{1}{2}(\epsilon+p) u^{\beta} \nabla_{\beta} \bar{\xi}^{\gamma} u^{\delta} \nabla_{\gamma} \xi_{\delta}-\frac{1}{2} p \nabla_{\alpha} \bar{\xi}_{\beta} \nabla^{\beta} \xi^{\alpha} \\
& +\frac{1}{2}(\epsilon+p)\left(u^{\beta} \nabla_{\alpha} \xi_{\beta} \bar{\xi}^{\gamma} \nabla_{\gamma} u^{\alpha}+u^{\alpha} \nabla_{\alpha} \xi_{\beta} \bar{\xi}^{\gamma} \nabla_{\gamma} u^{\beta}\right) \\
& +e^{-\nu} D_{\alpha}\left(e^{\nu} T^{\gamma[\alpha} \bar{\xi}^{\beta]} \nabla_{\beta} \xi_{\gamma}\right) .
\end{aligned}
$$

In this final equality, $D_{\alpha}$ is the derivative operator with respect to the 3 -metric on the $t=$ constant initial value surface. Then

$$
\begin{aligned}
\mathcal{E}_{f} \equiv & \mathcal{E}_{f 1}+\mathcal{E}_{f 2}+\mathcal{E}_{f 3} \\
= & \frac{1}{2}(\epsilon+p) u^{\beta} \nabla_{(\alpha} \xi_{\beta)} £_{\mathbf{u}} \bar{\xi}^{\alpha}-i \sigma(\epsilon+p) u^{t} \bar{\xi}_{\beta} u^{\gamma} \nabla_{\gamma} \xi^{\beta} \\
& +e^{-\nu} D_{\alpha}\left(e^{\nu} T^{\gamma[\alpha} \bar{\xi}^{\beta]} \nabla_{\beta} \xi_{\gamma}\right) \\
= & -\frac{i}{2}(\sigma-m \Omega)(\epsilon+p) u^{t} \bar{\xi}_{\beta} u^{\gamma} \nabla_{\gamma} \xi^{\beta}+\frac{i}{2}(\sigma+m \Omega)(\epsilon+p) u^{t} \bar{\xi}^{\alpha} \xi^{\beta} \nabla_{\alpha} u_{\beta} \\
& +e^{-\nu} D_{\alpha} A^{\alpha}
\end{aligned}
$$

where

$$
A^{\alpha}=e^{\nu}\left[T^{\gamma[\alpha} \bar{\xi}^{\beta]} \nabla_{\beta} \xi_{\gamma}+\frac{i}{2}(\sigma+m \Omega)(\epsilon+p) u^{t} \bar{\xi}^{\alpha} u^{\beta} \xi_{\beta}\right]
$$

The remaining term is

$$
\mathcal{E}_{g}=-\frac{1}{64 \pi} \epsilon^{t \gamma \delta \zeta} \epsilon^{t \epsilon \eta} £_{\mathbf{t}} \bar{h}_{\gamma \epsilon} £_{\mathbf{t}} h_{\delta \eta}=\frac{1}{64 \pi} e^{-2 \nu} e^{\alpha \beta} e^{\gamma \delta} £_{\mathbf{t}} \bar{h}_{\alpha \gamma} £_{\mathbf{t}} h_{\beta \delta}
$$

where $e_{\alpha \beta}$ is the metric on a 2 -sphere of radius $r$,

$$
e_{\alpha \beta}=r^{2}\left(\nabla_{\alpha} \theta \nabla_{\beta} \theta+\sin ^{2} \theta \nabla_{\alpha} \phi \nabla_{\beta} \phi\right)
$$

Thus

$$
E_{c}=\int d V\left(\mathcal{E}_{f}+\mathcal{E}_{g}\right)
$$

where the terms have opposite signs:

$$
\begin{aligned}
\mathcal{E}_{f}= & -\frac{1}{2}(m \Omega-\sigma)(m \Omega+\sigma) e^{-2 \nu}(\epsilon+p)|\xi|^{2} \\
& +2 m \Omega(\Omega-\omega) e^{-2 \nu}(\epsilon+p) r^{2} \sin \theta\left|\cos \theta \xi^{\theta} \xi^{\phi}\right|<0 \\
\mathcal{E}_{g}= & \frac{1}{64 \pi} e^{-2 \nu} e^{\alpha \beta} e^{\gamma \delta} \dot{\bar{h}}_{\beta \delta} \dot{h}_{\alpha \gamma}>0 .
\end{aligned}
$$


The fluid contribution $\mathcal{E}_{f}$ has the same form as the canonical energy (29) for Newtonian modes, from which the sign of (106) follows.

The sign of $E_{c}$ is negative because the fluid contribution is dominant. Writing

$$
\begin{aligned}
\mathcal{E}_{f}= & -\frac{2}{m+1}(\Omega-\omega)\left[\left(m \Omega-\frac{\Omega-\omega}{m+1}\right)-\cos ^{2} \theta\left(m^{2} \Omega+\frac{\Omega-\omega}{m+1}\right)\right] \times \\
& e^{-2 \nu}(\epsilon+p) \frac{\zeta^{2}}{r^{2}} \sin ^{2 m-2} \theta \\
\mathcal{E}_{g}= & 32 \pi\left[\frac{m}{(m+1)(m+2)}\right]^{2}(\Omega-\omega)^{2} \\
& e^{-2 \nu}(\epsilon+p)^{2} \zeta^{2} \sin ^{2 m} \theta\left(1+\frac{8 \cos ^{2} \theta}{\sin ^{4} \theta}\right)
\end{aligned}
$$

we have

$$
\frac{\int d \Omega \mathcal{E}_{g}}{\left|\int d \Omega \mathcal{E}_{f}\right|}=\frac{16}{[(m-1)(m+2)]^{2}} 2 \pi(\epsilon+p) r^{2}<2 \pi(\epsilon+p) r^{2} .
$$

The maximum value of $2 \pi(\epsilon+p) r^{2}$, for any equation of state, occurs for the maximum mass star. We directly calculate $2 \pi(\epsilon+p) r^{2}$ for the maximum mass star using equations of state spanning a wide range of stiffness. For equations of state $\mathrm{G}$ (excessively soft), C and L (excessively stiff) from the Arnett and Bowers (1977) catalogue we find

$$
2 \pi(\epsilon+p) r^{2}<0.4
$$

for all radius, implying

$$
E_{c}<0
$$

\section{Discussion}

Although a mode with negative canonical energy is unstable, the growth times of axial modes appear to be proportional to a high power of $\Omega$ for slowly rotating stars. Because the nonaxisymmetric instability of rotating perfect-fluid models is driven by gravitational radiation, its growth time for modes with a given $m$ is ordinarily shortest for modes that correspond to the smallest value of $l$ for a given $m$, namely $l=|m|$. For axial perturbations of slowly rotating stars, however, modes corresponding to a given $l$ and $m$ for the spherical star give rise to density perturbations associated with $Y_{l \pm 1}^{m}$. For nonisentropic stars, axial modes with $l=|m|+1$ give rise to $Y_{l}^{m}$ density perturbations, and as a result, these modes have the shortest growth time. However, if the star is isentropic, it can be shown (Provost et. al. (1981)) that the only axial normal modes of a Newtonian star have 
$l=|m|$, which contribute a $m+1$ multipole to the density perturbation. As a result, the gravitational radiation from mass multipoles is dominated by radiation from current multipoles (Lindblom, Owen \& Morsink 1998).

We provide here a rough estimate of the time scale for the axial instability of a mode with angular dependence $\exp (i m \phi)$, for a slowly rotating, nearly Newtonian model. A mode characterized by a displacement vector $\xi$ has energy of order

$$
E \sim-M \Omega^{2} \xi^{2}
$$

The mode is driven by gravitational radiation, with power dominated by the lowest order current multipole of order

$$
\frac{d E}{d t} \sim-\left(\frac{d^{l+1}}{d t^{l+1}} J_{l}^{m}\right)^{2} \sim-\Omega^{2 l+2}\left|J_{l}^{m}\right|^{2}
$$

where $J_{l}^{m}$ is the lowest current multipole perturbation of the star. We have

$$
J_{l}^{m} \sim \int \rho \delta v^{a} \epsilon_{a}^{b c} \nabla_{b} r \nabla_{c} Y_{l}^{m} r^{l} d V
$$

With our choice of $\xi, \delta v^{a}$ is $O(\Omega) \cdot ?$

$$
J_{l}^{m} \sim \Omega \xi R^{l+2}
$$

Finally, the growth time is of order

$$
\begin{aligned}
\tau & =E / \frac{d E}{d t}=\frac{M \Omega^{2} \xi^{2}}{\Omega^{2 m+2}\left|J_{m}^{m}\right|^{2}} \\
& \sim \frac{M}{(R \Omega)^{2 m+2}} .
\end{aligned}
$$

The precise calculation is given by Lindblom et. al. (1998). The timescale appears to have the same $\Omega$ dependence for relativistic stars (Friedman, Lockitch \& Morsink 1998).

The imaginary part of the frequency that Andersson (1997) computes is $O\left(\Omega^{2}\right)$, and our timescale estimate suggests that its magnitude is an artifact of an $O\left(\Omega^{2}\right)$ approximation method. But the sign of the imaginary part that his method obtains may correctly diagnose instability of a mode in the slow-rotation approximation.

\footnotetext{
${ }^{3}$ It would be more natural from a physical standpoint to take $\delta v^{a}=O(1), \xi=O\left(\Omega^{-1}\right)$ because as $\Omega \rightarrow 0$, the perturbation goes over to a nonvanishing, time-independent perturbation of the spherical star.
} 
The discussion so far has ignored viscosity, and, as in the case of polar modes, a long growth time for slow rotation implies that viscosity will enforce stability except for hot, rapidly rotating neutron stars (see Lindblom and Mendell 1995 and references therein). In particular, because the time-independent axial modes of a spherical star have nonvanishing shear, viscous damping must damp the instability for slow rotation.

For rapidly rotating stars, however, instability points have been computed numerically only for polar modes, initially for Newtonian models (Managan 1985, Imamura et al. 1985) and more recently for relativistic models in a Cowling approximation by Yoshida and Eriguchi (1997) and in the full theory by Stergioulas and Friedman (Stergioulas 1996, Stergioulas and Friedman 1997). Estimates of instability points when realistic values viscosity is included have similarly been restricted to polar perturbations. Extending these estimates to axial perturbations should then be straightforward. But for both axial and polar modes, a precise computation of instability points when viscosity is included is a more difficult task, apparently requiring the construction of outgoing modes and determination of their complex frequencies.

We are grateful to Nils Andersson for acquainting us with his results and for several

helpful discussions. This work was supported in part by NSF Grant No. PHY95-07740 and by NSERC of Canada.

\section{REFERENCES}

Andersson, N. 1997 "A new class of unstable modes of rotating relativistic stars," ApJ, to appear (gr-qc/9706075)

Arnett, W.D., \& Bowers, R. L. 1977, ApJ Sup., 33, 415

Chandrasekhar, S. 1970, Phys. Rev. Lett., 24, 611

Friedman, J. L., 1978, Commun. Math. Phys., 62, 247

Friedman, J. L., \& Ipser, J. R. 1992, Phil. Trans. R. Soc. Lond., A340, 391

Friedman, J.L., Lockitch, K.H., \& Morsink, S.M. 1998, paper in preparation

Friedman, J. L. \& Schutz, B. F. 1975, ApJ, 200, 204

Friedman, J. L \& Schutz, B. F. 1978, ApJ, 221, 937

Friedman, J. L. \& Schutz, B. F. 1978, ApJ, 222, 281

Imamura, J. N., Friedman, J. L. \& Durisen, R. H. 1985, ApJ, 294, 474 
Hartle, J.B., 1967, ApJ, 150, 1005

Lindblom, L. \& Mendell, G., 1995, ApJ, 444, 809

Lindblom, L., Owen, B.J., \& Morsink, S.M. 1998, Phys. Rev. Lett. 80, 4843 (gr-qc/9803053)

Managan, R. A. 1985, ApJ, 294, 463.

Papaloizou, J. \& Pringle, J. E., 1978, Mon. Not. R. astr. Soc., 182, 423.

Provost, J., Berthomieu, G. and Rocca, A., 1981, Astron. Astrophys. 94, 126.

Regge, T. \& Wheeler, J. A. 1957, Phys. Rev. 108, 1063

Stergioulas, N., 1996, The Structure and Stability of Rotating Relativistic Stars, Ph. D. Thesis, University of Wisconsin-Milwaukee.

Stergioulas, N. \& Friedman, J. L., 1998, ApJ, 492, 301

Yoshida, S. \& Eriguchi, Y. 1997, ApJ, 490, 779 